Página 10 - Acta Cirúrgica Brasileira - Vol. FALTA DE INFORMAČ̃̃O POR PARTE DOS 29 (Supl 2) 2014

\title{
Evaluation of antitumoral and
}

Development (CNPq) and Bahia Research Foundation (FAPESB)

LEIA-SE: antimicrobial activity of Morinda icitrifolia L. grown in Southeast Brazil ${ }^{1}$

National Council for Scientific and Technological Development (CNPq), Bahia Research Foundation (FAPESB) and ETENE/FUNDECI- Banco do Nordeste do Brasil

Thamyris CandidaI, Jerônimo Pereira de França"I, Alba Lucilvânia Fonseca Chaves"I, Fernanda Andrade Rodrigues Lopes $^{\text {III, Silvana Gaiba }}{ }^{\text {IV }}$, Celio Kersul do Sacramentov, Lydia Masako Ferreira ${ }^{\text {VI }}$, Lucimar Pereira de França ${ }^{I I}$

DOI: http://dx.doi.org/10.1590/S0102-86502014001400003

\section{5 - ORIGINAL ARTICLE}

\section{MODELS, BIOLOGICAL}

Evaluation of antimicrobial and antitumoral activity of Garcinia mangostana L. (mangosteen) grown in Southeast Brazil ${ }^{1}$

Bruna Lais Almeida Cunha', Jerônimo Pereira de França" Andrea Aparecida de Fátima Souza Moraes"II, Alba Lucilvânia Fonseca Chaves"I , Silvana Gaiba ${ }^{\text {Iv }}$, Renato Fontana", Celio Kersul do Sacramentov, Lydia Masako Ferreira $^{\mathrm{VI}}$, Lucimar Pereira de França ${ }^{\mathrm{II}}$

DOI: http://dx.doi.org/10.1590/S0102-86502014001400005
CORRIGENDA

Página 28 - Acta Cirúrgica Brasileira-Vol.

29 (Supl 2) 2014

\section{FALTA DE INFORMACÃO POR PARTE DOS}

AUTORES

ONDE SE LÊ:

Financial sources:

National Council for Scientific and Technological Development (CNPq) and Bahia Research Foundation (FAPESB)

LEIA-SE:

National Council for Scientific and Technological Development (CNPq), Bahia Research Foundation (FAPESB) and ETENE/FUNDECI- Banco do Nordeste do Brasil

Prof. Saul Goldenberg

Editor Responsável Acta Cir Bras 